\title{
Relação de dispersão para os plásmon-poláritons de superfície em uma interface plana metal/dielétrico
}

\author{
A. V. Andrade-Neto*1, Aroldo Ribeiro Neto ${ }^{2}$, Ado Jorio ${ }^{2}$ \\ ${ }^{1}$ Departamento de Física, Universidade Estadual de Feira de Santana, Feira de Santana, BA, Brasil \\ ${ }^{2}$ Departamento de Física, Universidade Federal de Minas Gerais, Belo Horizonte, MG, Brasil
}

Recebido em 14 de Novembro de 2016. Revisado em 13 de Fevereiro de 2017. Aceito em 14 de Fevereiro de 2017

\begin{abstract}
A plasmônica é uma disciplina emergente que investiga a interação entre a luz e os elétrons quase livres em sistemas metálicos em uma interface metal/dielétrico. Esta interação dá origem aos denominados Plásmon-Poláritons de Superfície SPPs (da sigla em inglês Surface Plasmon-Polaritons), que são um tipo de quasipartícula resultante do acoplamento entre o fóton e o plásmon de superfície. A relação de dispersão dos SPPs é um conceito chave no entendimento da interação da luz com a matéria em estruturas nanométricas, com aplicações diversas nos campos da fotônica e da bioengenharia. Neste trabalho apresentamos em detalhes a dedução da relação de dispersão para o plásmon-poláriton de superfície para uma interface plana metal/dielétrico. Para as partes real e imaginária do vetor de onda ao longo da interface, deduzimos expressões gerais cujos casos particulares reproduzem resultados conhecidos na literatura. Em seguida é realizada uma discussão sobre o significado físico dos resultados obtidos. Por fim, utilizando o modelo de Drude, apresentamos uma expressão explícita para a relação de dispersão na forma frequência versus vetor de onda.

Palavras-chave: plasmônica, plásmon de superfície, plásmon-poláriton de superfície.
\end{abstract}

Plasmonics is an emergent area of the nanophotonics that studies interaction between electromagnetic fields and free electrons in a metal on a metal/dielectric interface. Due to this interaction, the called surface plasmon polaritons (SPP) are generated. SPPs are electromagnetic excitations coupled to the collective oscillation of conduction electrons, which propagate along the interface between a conductor and a dielectric medium, with a broad range of applications, from photonics to bioengineering. One of the basic concepts related to SPPs is the dispersion relation, basic for understanding of the interaction between light and metallic nanostructures. In this paper we derive the dispersion relation of surface plasmon-polariton at planar interface and its fundamental properties are outlined.

Keywords: plasmonic, surface plasmon, surface plasmon polariton.

\section{Introdução}

A Física da Matéria Condensada, por sua grande amplitude de temas investigados, é considerada a mais ampla área da Física [1]. De um ponto de vista fundamental, os sistemas pesquisados em matéria condensada constituem problemas de muitos corpos de difícil solução, o que requer a utilização conjunta dos conceitos básicos de mecânica quântica, física estatística e eletromagnetismo. De um ponto de

\footnotetext{
*Endereço de correspondência: aneto@uefs.br
}

vista prático, muitos dos avanços tecnológicos que fazem parte do nosso cotidiano tiveram origem nos estudos em pesquisa básica nessa área. Podemos citar como exemplos a invenção do transistor, do laser de estado sólido, aparelhos de DVD, telas de cristal líquido, dentre muitos outros. Seus impactos não se limitam à engenharia de materiais, mas são responsáveis também pela inserção de novas tecnologias nas ciências da vida, como na regeneração de 
tecidos, terapias gênicas e antitumorais, e diversos outros avanços em bioengenharia 2].

A intensa investigação, tanto teórica quanto experimental, das propriedades eletrônicas, óticas, magnéticas, etc, que ocorrem na Física da Matéria Condensada possibilitou o desenvolvimento de novas disciplinas como a fotônica e a spintrônica. A plasmônica é uma subárea da nanofotônica que estuda a interação entre a radiação eletromagnética e os elétrons quase livres (elétrons de condução) em sistemas metálicos em uma interface metal/dielétrico. De forma mais pormenorizada, podemos dizer que a plasmônica lida com a geração, propagação e detecção de ondas de plasma ou ondas plasmônica em sistemas metálicos [3]. Vejamos o que exatamente significa essa afirmação.

Muitas propriedades físicas dos metais e semicondutores dopados podem ser entendidas em um contexto teórico no qual o comportamento dos elétrons de condução desses materiais se assemelha à de um plasma. Um plasma é um conjunto eletricamente neutro de partículas carregadas que interagem via forças coulombianas, no qual pelo menos um tipo é móvel e que exibe um comportamento coletivo [4]. Por analogia foi cunhada a expressão Plasma em Estado Sólido para descrever o comportamento dos elétrons de condução nesses materiais [5]. A neutralidade elétrica do sistema é garantida pela carga positiva da rede, cujo arranjo periódico discreto pode ser ignorado em uma primeira aproximação e tomada como contínua.

Esses elétrons apresentam uma oscilação coletiva cuja frequência, denominada frequência de plasma, é dada por (no sistema cgs) 6

$$
\omega_{p l}=\sqrt{\frac{4 \pi e^{2} n}{m^{*}}} .
$$

onde $e$ é a carga eletrônica, $m^{*}$ é a massa efetiva do elétron a qual incorpora a interação elétron-rede e $n$ é a concentração dos elétrons de condução.

Segundo a teoria quântica de campos é possível quantizar um campo oscilatório e associar ao mesmo um quantum elementar ao qual se pode atribuir propriedades de partícula [7. Por exemplo, os fótons são as partículas (quantum elementar) associadas ao campo eletromagnético (uma interessante discussão sobre o conceito de fóton é encontrada na Ref. [8]). O quantum associado às oscilações coletivas de um gás de elétrons é denominado de plásmon. O plásmon é um exemplo das denominadas quasipartículas (ou excitações elementares) as quais, de forma simplificada, podem ser definidas como entidades fictícias que descrevem de modo relativamente simples o comportamento de sistemas de muitos corpos em matéria condensada 9,10 . A energia de um plásmon é calculada pela relação

$$
E=\hbar \omega_{p l}
$$

onde $\hbar$ é a constante de Planck normalizada. Para metais, a concentração dos elétrons de condução é $n \sim 10^{22} \mathrm{~cm}^{-3}$, o que implica em uma energia do plásmon da ordem de $10 \mathrm{eV}$. Para um semicondutor, de grande volume (bulk), considerando $n \sim 10^{18}$ $\mathrm{cm}^{-3}$, teremos $\hbar \omega_{p l}$ da ordem de $0,01 \mathrm{eV}$ (este valor pode mudar significativamente em materiais de baixa dimensionalidade). Classicamente, o plásmon corresponde a uma oscilação na densidade de carga eletrônica e esta oscilação pode ocorrer no interior ou na superfície do material. No primeiro caso falamos em plásmons de volume (bulk plasmons) ou simplesmente plásmons. No segundo caso falamos em plásmons de superfície (surface plasmons).

Outro exemplo de quasipartícula que nos interessa neste trabalho é o poláriton, o qual resulta do acoplamento entre o fóton e uma excitação elementar do sólido. Há vários tipos de poláritons, a depender da quasiparticula que se acopla com o fóton. Fônon-poláriton que resulta do acoplamento entre o fônon transversal ótico e o fóton; o éxcitonpoláriton que resulta do acoplamento entre o éxciton (par elétron-buraco ligado) e o fóton e, finalmente, o plásmon-poláriton de volume - BPP (da acrossemia Bulk Plasmon Polariton) que resulta da interação entre os fótons e os elétrons livres no material. No presente trabalho estamos interessados neste último. Vale a pena observar que a denominação plásmonpoláriton não é muito adequada pois pode induzir o leitor a imaginar que exista um acoplamento entre o plásmon e o fóton. Conforme é discutido no Apêndice $\mathrm{A}$, não pode haver acoplamento entre o fóton e o plásmon de volume já que este representa uma oscilação longitudinal, enquanto o fóton repesenta uma oscilação transversal. Contudo, em uma interface em que incida uma onda eletromagnética p-polarizada, a polarização pode ser decomposta em uma componente perpendicular à interface e outra componente paralela à interface. A componente paralela torna-se uma onda longitudinal e, desse modo, pode acoplar-se com o plásmon de superfície. Esse acoplamento dá origem ao Plásmon-Poláriton 
de Superfície (SPP da acrossemia Surface Plasmon Polaritons).

Do ponto de vista da física clássica, os PlásmonPoláritons de Superfície podem ser visualizados como uma onda eletromagnética superficial que se propaga na interface entre o metal e o dielétrico e que decai exponencialmente com a distância na direção perpendicular à interface, provocando o confinamento da luz na interface metal/dielétrico. Esse efeito de confinamento pode ser utilizado em uma grande variedades de aplicações tais como na fabricação de LEDs mais eficientes, no aumento da resolução de microscópios e na melhora da sensibilidade de detectores químicos e biológicos 11. Uma das mais interessantes aplicações da plasmônica ocorre na denominada microscopia de campo próximo. Com o advento do Microscópio Ótico de Campo Próximo SNOM (da sigla em inglês Scanning Near-Field Optical Microscope) é possivel obter imagens com uma resolução muito maior do que a estabelecida pelo critério de Rayleigh [12], o qual, devido ao fenômeno de difração da luz [13], determina o limite da resolução espacial de um sistema ótico convencional a aproximadamente metade do comprimento de onda da luz utilizada como prova. A grande vantagem do SNOM é que ele combina a grande resolução dos Microscópios de Varredura por Sonda SPM (da sigla em inglês Scanning Probe Microscope) com a versatilidade das técnicas óticas [6, 14].

O estudo dos Plásmon-Poláritons de Superfície fornece importante informações sobre o comportamento da luz em superfícies metálicas de tamanhos nanométricos. Esse comportamento pode ser investigado a partir da análise das equações de Maxwell e suas apropriadas condições de contorno. Este é o caminho que seguiremos neste trabalho. Em particular, deduziremos a relação de dispersão para os PlásmonPoláritons de Superfície em uma interface plana metal/dielétrico e exploraremos as informações físicas que podem ser obtidas a partir dessa relação.

\section{Dedução da relação de dispersão dos plásmon-poláritons de superfície em uma interface metal-dielétrico plana}

As propriedades dos plásmon-poláritons de superfície na interface entre um metal e um dielétrico podem ser obtidas a partir das equações de Maxwell em cada meio e das condições de contorno asso- ciadas ao campo eletromagnético na superfície de descontinuidade entre os dois meios.

As condições de contorno sobre os vetores do campo eletromagnético nos informam como esses se comportam na interface entre dois meios com propriedades materiais diferentes. Este é um problema abordado nos livros textos de eletromagnetismo [ver, por exemplo, Ref. [15]]. Mostraremos a seguir que, partindo das soluções das equações de Maxwell e das condições de contorno, podemos obter a relação de dispersão para o SPP em uma interface plana metal dielétrico e dessa relação inferir as propriedades físicas do sistema.

Vamos considerar dois meios semi-infinitos separados por uma interface plana. O meio 1 é um metal caracterizado por uma função dielétrica complexa dependente da frequência $\omega, \epsilon_{1}(\omega)=\epsilon_{1}^{\prime}(\omega)+\imath \epsilon_{1}^{\prime \prime}(\omega)$ onde $\epsilon_{1}^{\prime}(\omega)$ e $\epsilon_{1}^{\prime \prime}(\omega)$ representam as partes real e imaginária, respectivamente. Por sua vez, para o meio 2 , meio dielétrico, assumiremos que a função dielétrica $\epsilon_{2}$ é uma grandeza real e constante. Vamos escolher um sistema de coordenadas cartesianas onde $(x, z)$ é o plano de incidência e a interface coincide com o plano $z=0$, conforme esquematizado na Figura 1.

Os campos de uma onda plana monocromática podem ser representados como superposições de duas polarizações lineares perpendiculares que, por conveniência, são escolhidas como perpendicular ao plano de incidência (denominado s-polarizado) e outra paralela ao plano de incidência (denominado p-polarizado). É possível mostrar [16] que a componente perpendicular (onda s-polarizada) não produz carga superficial. Assim, podemos considerar apenas ondas p-polarizada, cujas expressões para os campos elétrico e magnético podem ser escritas como 17

$$
\vec{E}_{1}=\left(E_{01 x} \hat{x}+E_{01 z} \hat{z}\right) \exp \left[\imath\left(k_{1 x} x-k_{1 z} z-\omega t\right)\right]
$$

$$
\vec{H}_{1}=\left(H_{01 y} \hat{y}\right) \exp \left[\imath\left(k_{1 x} x-k_{1 z} z-\omega t\right)\right],
$$

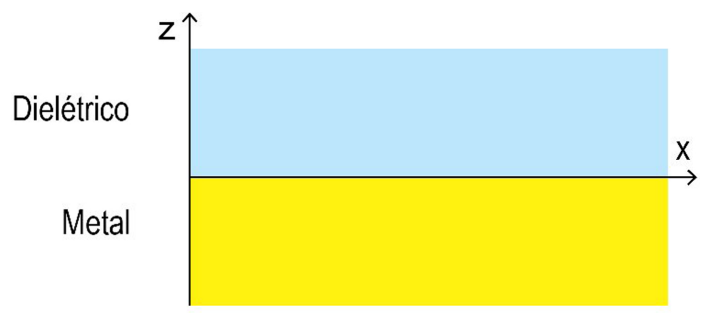

Figura 1: Esquema geométrico para uma interface metal/dielétrico no plano $z=0$. O meio dielétrico ocupa o semi-espaço $z>0$ enquanto o metal o semi-espaço $z<0$. 
para $z<0$

$$
\begin{gathered}
\vec{E}_{2}=\left(E_{02 x} \hat{x}+E_{02 z} \hat{z}\right) \exp \left[\imath\left(k_{2 x} x+k_{2 z} z-\omega t\right)\right] \\
\vec{H}_{2}=\left(H_{02 y} \hat{y}\right) \exp \left[\imath\left(k_{2 x} x+k_{2 z} z-\omega t\right)\right]
\end{gathered}
$$

para $z>0$, onde $E_{0 x}$ é a componente $x$ da amplitude do campo elétrico, $k_{x}$ é a componente $x$ do vetor de onda e $\hat{x}$ representa o vetor unitário na direção do eixo $x$. Idem para as outras componentes. As equações (3) 6 ) podem ser escritas de forma mais compacta e mais adequada para manipulação como

$$
\begin{gathered}
\vec{E}_{j}=E_{j x} \hat{x}+E_{j z} \hat{z} \\
\vec{H}_{j}=H_{j y} \hat{y},
\end{gathered}
$$

onde o índice $j$ identifica o meio: $j=1$ indica o meio metálico e $j=2$ o meio dielétrico com

$$
\begin{aligned}
& E_{j x}=E_{0 j x} e^{\imath \varphi_{j}} \\
& E_{j z}=E_{0 j z} e^{\imath \varphi_{j}} \\
& H_{j y}=H_{0 j y} e^{\imath \varphi_{j}}
\end{aligned}
$$

e

$$
\varphi_{j}=k_{j x} x \mp k_{j z} z-\omega t
$$

com o sinal - para $j=1$ e o sinal + para $j=2$.

As equações de Maxwell em um meio material não-magnético, na ausência de fontes, em termos de componentes de Fourier são escritas como (ver detalhes no Apêndice B)

$$
\begin{gathered}
\nabla \times \vec{H}_{j}(\vec{r}, \omega)=-\frac{\imath \omega}{c} \epsilon_{j}(\omega) \vec{E}_{j}(\vec{r}, \omega) \\
\nabla \times \vec{E}_{j}(\vec{r}, \omega)=\frac{\imath \omega}{c} \vec{H}_{j}(\vec{r}, \omega) \\
\nabla \cdot\left(\epsilon_{j}(\omega) \vec{E}_{j}(\vec{r}, \omega)\right)=0 \\
\nabla \cdot \vec{H}_{j}(\vec{r}, \omega)=0
\end{gathered}
$$

onde, mais uma vez, o índice $j=1$ ou $j=2$ identifica o meio.

Devemos agora indicar como os vetores de campo variam na interface entre os dois meios. A componente tangencial do campo elétrico $\vec{E}$ é contínua na interface entre dois meios, bem como a componente normal do vetor deslocamento $\vec{D}$ se não há cargas superficiais livres, como acontece no presente caso. Do mesmo modo, a componente normal do campo magnético $\vec{H}$ é contínua através da interface 15 . Utilizando essas condições de contorno obtemos

$$
\begin{gathered}
E_{01 x}=E_{02 x} ; \\
H_{01 y}=H_{02 y} ; \\
\epsilon_{1} E_{01 z}=\epsilon_{2} E_{02 z} .
\end{gathered}
$$

Utilizando a equação 17 vemos que a componente $x$ do vetor de onda é contínua, i.e.,

$$
k_{1 x}=k_{2 x}=k_{x} .
$$

Vamos utilizar as equações de Maxwell e as condições de contorno para deduzir a relação de dispersão para o plásmon-poláriton de superfície. Aplicando o rotacional na equação (8) temos que

$$
\nabla \times \vec{H}_{j}=-\frac{\partial H_{j y}}{\partial z} \hat{x}+\frac{\partial H_{j y}}{\partial x} \hat{z} .
$$

Utilizando as equações (7) e (21) em (13) temos que

$$
-\frac{\partial H_{j y}}{\partial z} \hat{x}+\frac{\partial H_{j y}}{\partial x} \hat{z}=-\frac{\imath \omega}{c} \epsilon_{j}\left(E_{j x} \hat{x}+E_{j z} \hat{z}\right) .
$$

Utilizando as equações (9 11) em (22) obtemos explicitamente para os meios 1 e 2 :

Para o meio 1

$$
\begin{aligned}
& k_{1 x} H_{01 y}=-\frac{\omega}{c} \epsilon_{1}(\omega) E_{01 z} ; \\
& k_{1 z} H_{01 y}=-\frac{\omega}{c} \epsilon_{1}(\omega) E_{01 x} .
\end{aligned}
$$

Para o meio 2

$$
\begin{gathered}
k_{2 x} H_{02 y}=-\frac{\omega}{c} \epsilon_{2} E_{02 z} ; \\
k_{2 z} H_{02 y}=\frac{\omega}{c} \epsilon_{2} E_{02 x} .
\end{gathered}
$$

Das equações (17), 24) e 26 obtemos

$$
k_{1 z} H_{01 y}=-\frac{\epsilon_{1}}{\epsilon_{2}} k_{2 z} H_{02 y}
$$

a qual, junto com a Eq. (18), forma o sistema 


$$
\left\{\begin{array}{l}
H_{01 y}-H_{02 y}=0 \\
\frac{k_{z 1}}{\epsilon_{1}(\omega)} H_{01 y}+\frac{k_{z 2}}{\epsilon_{2}} H_{02 y}=0 .
\end{array}\right.
$$

Para que o sistema acima tenha solução não trivial o determinante tem que ser igual a zero. Assim, obtemos

$$
\frac{k_{z 1}}{\epsilon_{1}(\omega)}+\frac{k_{z 2}}{\epsilon_{2}}=0
$$

De forma semelhante, partindo da equação (14) obtemos após alguns cálculos

$$
k_{x}^{2}+k_{j z}^{2}=\left(\frac{\omega}{c}\right)^{2} \epsilon_{j} .
$$

Das equações (29) e (30) obtemos a relação de dispersão

$$
k_{x}(\omega)=\frac{\omega}{c} \sqrt{\frac{\epsilon_{1}(\omega) \epsilon_{2}}{\epsilon_{1}(\omega)+\epsilon_{2}}} .
$$

A equação (31) expressa a relação entre o vetor de onda ao longo da interface $k_{x}$ e a frequência angular $\omega$ e é denominada relação de dispersão para os plásmon-poláritons de superfície em uma interface entre um metal e um dielétrico. Essa equação é fundamental para o entendimento da interação

entre a luz e a matéria em uma interface metaldielétrico, que dá origem ao plásmon-poláriton de superfície. Suas implicações físicas serão discutidas nas próximas Seções.

Deve ser observado que a expressão (31) foi deduzida utilizando apenas as equações de Maxwell e, portanto, ela é geral. Como $\epsilon_{1}(\omega)$ é uma função complexa, $k_{x}$ é também uma função complexa, i.e., $k_{x}=k_{x}^{\prime}+\imath k_{x}^{\prime \prime}$, cujas expressões gerais serão deduzidas a seguir.

\section{Vetor de onda dos plásmon-poláritons de superfície: caso geral}

Considerando que $\epsilon_{1}(\omega)=\epsilon_{1}^{\prime}(\omega)+\imath \epsilon_{1}^{\prime \prime}(\omega)$ a equação (31) pode ser reescrita como

$$
k_{x}^{\prime}+\imath k_{x}^{\prime \prime}=\sqrt{Z^{\prime}(\omega)+\imath Z^{\prime \prime}(\omega)},
$$

onde

$$
\begin{array}{r}
Z^{\prime}(\omega)=\frac{\epsilon_{1}^{\prime}(\omega) \epsilon_{2}\left[\epsilon_{1}^{\prime}(\omega)+\epsilon_{2}+\epsilon_{1}^{\prime \prime}(\omega)\right]}{\left[\epsilon_{1}^{\prime}(\omega)+\epsilon_{2}\right]^{2}+\left[\epsilon_{1}^{\prime \prime}(\omega)\right]^{2}}, \\
Z^{\prime \prime}(\omega)=\frac{\epsilon_{2}^{2} \epsilon_{1}^{\prime \prime}(\omega)}{\left[\epsilon_{1}^{\prime}(\omega)+\epsilon_{2}\right]^{2}+\left[\epsilon_{1}^{\prime \prime}(\omega)\right]^{2}} .
\end{array}
$$

Da equação (32) obtemos que

$$
\begin{gathered}
\left(k_{x}^{\prime}\right)^{2}=\left(\frac{\omega}{c}\right)^{2}\left[\frac{Z^{\prime}(\omega)+\sqrt{\left[Z^{\prime}(\omega)\right]^{2}+\left[Z^{\prime \prime}(\omega)\right]^{2}}}{2}\right], \\
\left(k_{x}^{\prime \prime}\right)^{2}=\left(\frac{\omega}{c}\right)^{4} \frac{\left[Z^{\prime \prime}(\omega)\right]^{2}}{4\left(k_{x}^{\prime}\right)^{2}}=\left(\frac{\omega}{c}\right)^{2}\left[\frac{\left[Z^{\prime \prime}(\omega)\right]^{2}}{2\left(Z^{\prime}(\omega)+\sqrt{\left[Z^{\prime}(\omega)\right]^{2}+\left[Z^{\prime \prime}(\omega)\right]^{2}}\right)}\right] .
\end{gathered}
$$

Para os metais nobres em geral, temos que a parte real da função dielétrica é negativa e a parte imaginária é positiva, i.e., $\epsilon_{1}^{\prime}<0$ e $\epsilon_{1}^{\prime \prime}>0$. Para os dielétricos $\epsilon_{2}>0$. Nessas condições vemos da Eq. (34) que $Z^{\prime \prime}$ é sempre uma grandeza real positiva. Por sua vez, $Z^{\prime}$ pode ser positiva, nula ou negativa. Se $\left|\epsilon_{1}^{\prime}\right|>\epsilon_{2}+\epsilon_{1}^{\prime \prime}, Z^{\prime}>0$. Se $\left|\epsilon_{1}^{\prime}\right|=\epsilon_{2}+\epsilon_{1}^{\prime \prime}$, isso implica em $Z^{\prime}=0$. Finalmente, se $\left|\epsilon_{1}^{\prime}\right|<\epsilon_{2}+\epsilon_{1}^{\prime \prime}$, vemos que $Z^{\prime}<0$. Contudo, qualquer que seja o caso, $k_{x}^{\prime}$ e $k_{x}^{\prime \prime}$ são números reais positivos.

As expressões gerais (35) e (36) são suficientementes complicadas para que valha a pena analisar alguns casos limites em que elas se reduzem a formas mais simples conhecidas da literatura. Em geral é considerada a situação em que a parte imaginária da função dielétrica do metal é muito menor que o módulo da parte real, i.e., $\epsilon_{1}^{\prime \prime}<<\left|\epsilon_{1}^{\prime}\right|$. Neste limite vemos que

$$
Z^{\prime \prime}<<Z^{\prime} \approx\left(\frac{\omega}{c}\right)^{2} \frac{\epsilon_{1}^{\prime}(\omega) \epsilon_{2}}{\epsilon_{1}^{\prime}(\omega)+\epsilon_{2}} .
$$

\section{Então obtemos}




$$
k_{x}^{\prime}(\omega) \approx \frac{\omega}{c} \sqrt{\frac{\epsilon_{1}^{\prime}(\omega) \epsilon_{2}}{\epsilon_{1}^{\prime}(\omega)+\epsilon_{2}}} .
$$

Do mesmo modo obtemos da equação (36)

$$
k_{x}^{\prime \prime}(\omega) \approx \frac{1}{2} \frac{\omega}{c}\left(\frac{\epsilon_{1}^{\prime}(\omega) \epsilon_{2}}{\epsilon_{1}^{\prime}(\omega)+\epsilon_{2}}\right)^{3 / 2} \frac{\epsilon_{1}^{\prime \prime}}{\left(\epsilon_{1}^{\prime}(\omega)\right)^{2}} .
$$

As equações (38) e (39) são as expressões normalmente encontradas na literatura 6 .

\section{Significado físico dos resultados obtidos}

Vamos agora iniciar uma discussão sobre o significado físico dos resultados obtidos nas seções anteriores.

\subsection{Comprimento de onda e comprimento de propagação dos plásmon-poláritons de superfície}

Utilizando (20), vemos das expressões (3) e (5) que o campo elétrico na interface é da forma

$$
\vec{E}_{j}=\left(E_{0 j x} \hat{x}+E_{0 j z} \hat{z}\right) \exp \left[\imath\left(k_{x}^{\prime}+\imath k_{x}^{\prime \prime}\right) x\right] e^{-\imath \omega t} .
$$

onde $k_{x}^{\prime}$ e $k_{x}^{\prime \prime}$ são dados, respectivamente, pelas equações (35) e (36) no caso geral, ou (38) e (39) no caso particular $\epsilon_{1}^{\prime \prime}<<\left|\epsilon_{1}^{\prime}\right|$. A equação $\sqrt{40}$ representa um campo elétrico progressivo amortecido.

A parte real do vetor de onda, $k_{x}^{\prime}$, determina o comprimento de onda do SPP, $\lambda_{S P P}=2 \pi / k_{x}^{\prime}$, o qual no caso limite $\epsilon_{1}^{\prime \prime}<<\left|\epsilon_{1}^{\prime}\right|$ é dado por

$$
\lambda_{S P P}(\omega)=\lambda_{o} \sqrt{\frac{\epsilon_{1}^{\prime}(\omega)+\epsilon_{2}}{\epsilon_{1}^{\prime}(\omega) \epsilon_{2}}} .
$$

onde $\lambda_{o}=(2 \pi c) / \omega$ é o comprimento de onda da luz no vácuo. Nas regiões do visível e infravermelho, para metais nobres e um dielétrico típico, temos que $\left|\epsilon_{1}^{\prime}\right|>\epsilon_{2}$. Como pode ser visto da equação 41 , isso implica que

$$
\frac{\lambda_{S P P}}{\lambda_{o}}<1
$$

Vemos então que $\lambda_{S P P}$ é sempre menor que o comprimento de onda da radiação que excita o sistema. Esse fato é a base que possibilita a construção de dispositivos óticos (circuitos plasmônicos) em tamanhos menores que o comprimento de onda da luz visível.

Já a parte imaginária, $k_{x}^{\prime \prime}$, determina o comprimento de propagação do SPP quando este se propaga ao longo da interface. Vemos da equação 40 que $k_{x}^{\prime \prime}$ produz um decaimento exponencial da amplitude do campo elétrico na interface. Isso nos permite definir um comprimento de propagação para o SPP, que é a distância para a qual o campo elétrico decai $1 / e$ do seu valor inicial, ou seja, $L_{S P P}=1 / k_{x}^{\prime \prime}$ (ou $L_{S P P}=1 / 2 k_{x}^{\prime \prime}$ para a intensidade). Usando a equação (39) obtemos

$$
L_{S P P}(\omega)=\frac{\lambda_{o}}{\pi}\left[\frac{\epsilon_{1}^{\prime}(\omega)+\epsilon_{2}}{\epsilon_{1}^{\prime}(\omega) \epsilon_{2}}\right]^{3 / 2} \frac{\left[\epsilon_{1}^{\prime}(\omega)\right]^{2}}{\epsilon_{1}^{\prime \prime}(\omega)} .
$$

O comprimento de propagação $L_{S P P}$ define um comprimento máximo para um circuito plasmônico. A Figura 2 mostra o comprimento de propagação do SPP em função do comprimento de onda, calculado pela equação (43), para uma interface ar/prata. Para calcular a função dielétrica utilizamos o modelo de Drude, ver equações (52) e (53) adiante, com os parâmetros $\hbar \omega_{p l}=7.9 \mathrm{eV}$ e $\hbar \Gamma=0.06 \mathrm{eV}$. Vemos que o SPP pode percorrer uma grande distância (considerando dispositivos na escala nanométrica) antes de se dispersar.

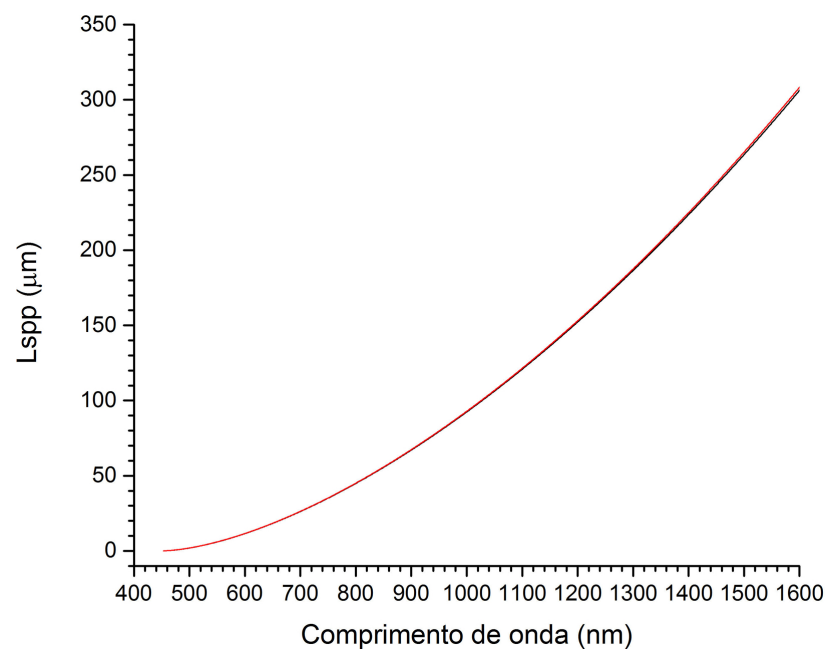

Figura 2: Comprimento de propagação $L_{S P P}$ para uma interface ar/prata, usando o modelo de Drude para o cálculo da função dielétrica com os parâmetros $\hbar \omega_{p l}=7.9 \mathrm{eV}$ e $\hbar \Gamma=0.06 \mathrm{eV}$.

\subsection{Profundidade de penetração dos plásmon-poláritons de superfície}

$\mathrm{Na}$ direção perpendicular à interface, o campo elétrico decai exponencialmente com a distância 
a interface nos dois meios. De fato, se substituimos a equação (31) na equação (30) obtemos

$$
k_{j z}=\frac{\omega}{c} \sqrt{\frac{\epsilon_{j}^{2}}{\epsilon_{1}+\epsilon_{2}}} .
$$

Como para metais típicos $\epsilon_{1}+\epsilon_{2}<0$, vemos que $k_{j z}$ é uma grandeza imaginária. Para o caso limite em que $\epsilon_{1}^{\prime \prime}<<\left|\epsilon_{1}^{\prime}\right|$ obtemos para o meio 1

$$
k_{1 z}=\frac{\omega}{c} \sqrt{\frac{\left(\epsilon_{1}^{\prime}\right)^{2}}{\epsilon_{1}^{\prime}+\epsilon_{2}}}=\imath\left|k_{1 z}\right|,
$$

onde

$$
\left|k_{1 z}\right|=\frac{\omega}{c} \sqrt{\frac{\left(\epsilon_{1}^{\prime}\right)^{2}}{\left|\epsilon_{1}^{\prime}\right|-\epsilon_{2}}} .
$$

Para o meio 2 temos que

$$
k_{2 z}=\frac{\omega}{c} \sqrt{\frac{\epsilon_{2}^{2}}{\epsilon_{1}^{\prime}+\epsilon_{2}}}=\imath\left|k_{2 z}\right|,
$$

onde

$$
\left|k_{2 z}\right|=\frac{\omega}{c} \sqrt{\frac{\epsilon_{2}^{2}}{\left|\epsilon_{1}^{\prime}\right|-\epsilon_{2}}} .
$$

Utilizando as equações (45) e (47), as equações (5) e (3) podem ser rescritas como

$$
\vec{E}_{j}=\left(E_{0 j x} \hat{x}+E_{0 j z} \hat{z}\right) \exp \left[\imath\left(k_{x} x-\omega t\right)\right] e^{-\left|k_{j z}\right||z|},
$$

que representa uma onda que se propaga na direção $x$ e decai exponencialmente na direção $z$. Esse tipo de onda é denominada onda evanescente.

A profundidade de penetração é definida como a distância $l=|z|$ para a qual o campo eletromagnético se reduz a $1 / e$ do seu valor na interface. Assim

$$
l_{j}=\frac{1}{\left|k_{j z}\right|} .
$$

Para uma interface ar/prata, para comprimentos de onda no intervalo entre 500 a $1500 \mathrm{~nm}$, a profundidade de penetração no ar, $l_{2}$, está situada na faixa entre 450 a $2500 \mathrm{~nm}$. Por sua vez, para o mesmo intervalo de comprimentos de onda, a profundidade de penetração na prata será $l_{1} \sim 26 \mathrm{~nm}$, sendo praticamente independente do comprimento de onda 3 . Isso mostra que a profundidade de penetração no meio metálico é muito menor que no meio dielétrico.

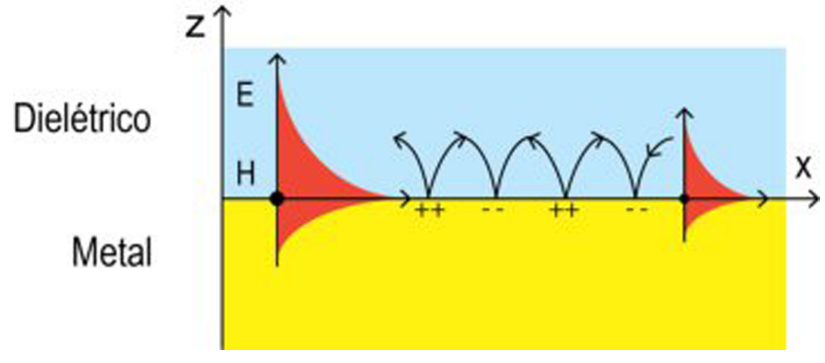

Figura 3: Esquema da distribuição de carga na interface metal/dielétrico. Também é esquematizado (em vermelho) o decaimento exponencial dos campos elétricos nos meios metálico e dielétrico, perpendicularmente à superfície da interface. As linhas curvas com setas indicam as linhas de força do campo elétrico.

A Figura 3 mostra de forma esquemática a oscilação de carga elétrica na interface metal/dielétrico. Deve ser observado que as cargas positivas indicam região na qual há uma diminuição de carga negativa e não um excesso de carga positiva. Também são representadas as linhas de força do campo elétrico nas proximidades da interface (linhas curvas com setas) bem como a profundidade de penetração nos dois meios (em vermelho).

\subsection{Relação de dispersão na forma $\omega=\omega\left(k_{x}^{\prime}\right)$}

Uma relação de dispersão é representada, em geral, como uma relação entre a energia, expressa em termos da frequência angular $\omega$, e o momento linear, expresso em termos do vetor de onda $k_{x}^{\prime}$. Queremos obter, para o SPP, uma equação mais explícita do tipo $\omega=\omega\left(k_{x}^{\prime}\right)$. Para isso, vamos utilizar o modelo de Drude, o qual, apesar de sua simplicidade, fornece uma boa descrição das propriedades óticas e de transporte de metais e semicondutores dopados. Neste modelo a interação entre os elétrons de condução do material é negligenciada e os mesmos são espalhados de maneira aleatória pelos íons da rede com uma frequência característica $\Gamma=1 / \tau$, onde $\tau$ é um tempo de relaxação médio [19]. O modelo de Drude fornece a seguinte expressão para a função dielétrica de um gás de elétrons [19]

$$
\epsilon_{1}(\omega)=\epsilon_{\infty}-\frac{\omega_{p l}^{2}}{\omega(\omega+\imath \Gamma)}
$$

onde $\epsilon_{\infty}$ é a constante dielétrica a altas frequência do material, $\omega_{p l}$ é a frequência de plasma do gás de elétrons dada pela Eq.(1) e $\Gamma$, como definido acima, representa uma constante de amortecimento. 
Separando a equação acima nas suas partes real e imaginária obtemos

$$
\begin{gathered}
\epsilon_{1}^{\prime}(\omega)=\epsilon_{\infty}-\frac{\omega_{p l}^{2}}{\omega^{2}+\Gamma^{2}}, \\
\epsilon_{1}^{\prime \prime}(\omega)=\frac{\omega_{p l}^{2} \Gamma}{\omega\left(\omega^{2}+\Gamma^{2}\right)} .
\end{gathered}
$$

Para metais típicos temos que $\omega_{p l} \gg \Gamma$. Para a prata, por exemplo, temos que $\hbar \omega_{p l}=7.9 \mathrm{eV}$ e $\hbar \Gamma=0.06 \mathrm{eV}[18]$. Isso significa que podemos considerar apenas a parte real da função dielétrica para a obtenção da relação de dispersão. Usando a equação (52) na equação (38) temos que

$$
k_{x}^{\prime}=\frac{\omega}{c} \sqrt{\frac{\epsilon_{1}^{\prime} \epsilon_{2}}{\epsilon_{1}^{\prime}+\epsilon_{2}}}=\frac{\omega}{c} \sqrt{\frac{\epsilon_{2}\left[\epsilon_{\infty}\left(\omega^{2}+\Gamma^{2}\right)-\omega_{p l}^{2}\right]}{\left(\epsilon_{\infty}+\epsilon_{2}\right)\left(\omega^{2}+\Gamma^{2}\right)-\omega_{p l}^{2}}}
$$

Podemos reescrever a equação 54 na seguinte forma

$$
\begin{aligned}
& \omega^{2}\left(k_{x}^{\prime}\right)=\frac{1}{2}\left[\frac{\omega_{p l}^{2}}{\epsilon_{\infty}}+\frac{\left(\epsilon_{\infty}+\epsilon_{2}\right)}{\epsilon_{\infty} \epsilon_{2}} c^{2}\left(k_{x}^{\prime}\right)^{2}-\Gamma^{2}\right] \\
& -\frac{1}{2} \sqrt{\left(\frac{\omega_{p l}^{2}}{\epsilon_{\infty}}+\frac{\left(\epsilon_{\infty}+\epsilon_{2}\right)}{\epsilon_{\infty} \epsilon_{2}} c^{2}\left(k_{x}^{\prime}\right)^{2}-\Gamma^{2}\right)^{2}-4 c^{2}\left(k_{x}^{\prime}\right)^{2}\left[\frac{\left.\omega_{p l}^{2}-\left(\epsilon_{\infty}+\epsilon_{2}\right) \Gamma^{2}\right)}{\epsilon_{\infty} \epsilon_{2}}\right]}
\end{aligned}
$$

Se o meio dielétrico for o vácuo $\left(\epsilon_{2}=1\right)$ e fazendo $\Gamma=0$ e $\epsilon_{\infty}=1$ a equação 55 torna-se

$$
\omega^{2}\left(k_{x}^{\prime}\right)=\frac{\omega_{p l}^{2}}{2}+c^{2}\left(k_{x}^{\prime}\right)^{2}-\sqrt{\frac{\omega_{p l}^{4}}{4}+c^{4}\left(k_{x}^{\prime}\right)^{4}},
$$

que representa a relação de dispersão para o SPP. De forma semelhante, podemos obter a relação de dispersão para o plásmon-polaríton de volume. Da condição de transversalidade da onda eletromagnética, obtemos das equações de Maxwell a seguinte expressão, conforme equação A.6 do Apêndice A,

$$
\left(k_{x}^{\prime}\right)^{2}=\frac{\omega^{2}}{c^{2}} \epsilon(\omega) .
$$

Utilizando (52) em (57), e considerando $\Gamma=0$, obtemos a relação de dispersão para o plásmonpoláriton de volume.

$$
\omega^{2}\left(k_{x}^{\prime}\right)=\omega_{p l}^{2}+c^{2}\left(k_{x}^{\prime}\right)^{2} .
$$

Na Figura 4 são mostradas as relações de dispersão para o plásmon-poláriton de volume (BPP) e para o plásmon-poláriton de superfície (SPP) em uma interface metal dielétrico. O eixo da frequência está normalizado pela frequência de plasma, enquanto o eixo horizontal está em unidades de $k_{x}^{\prime} c / \omega_{p l}$. A relação de dispersão para o BPP está representada pela curva em vermelho (obtida da equação (58)). Por sua vez, a relação de dispersão do SPP, obtida da equação (56), é a curva em preto. A relação de dispersão para a luz no vácuo (linha verde) é dada pela relação linear, $\omega=c k_{x}^{\prime}$ e é denominada linha da luz. Finalmente, a linha azul representa a relação de dispersão para o plasmon de superfície $\left(\omega=\omega_{S}=\omega_{p l} / \sqrt{2}\right)$ e é denominada linha do plasmon de superfície.

Muitas das propriedades físicas dos SPPs podem ser entendidas a partir da análise da Figura 4, conforme faremos a seguir.

Fisicamente, o SPP pode ser intepretado como sendo o resultado do acoplamento entre as oscilações coletivas dos elétrons na superfície (cujos quanta são os plásmons de superfície) e uma onda eletromagnética superficial (cujos quanta são os fótons). $\mathrm{Na}$ interface metal/dielétrico ocorre uma forte interação entre os modos fotônico e plasmônico representados, respectivamente, pela linha de luz e linha de plasmon de superfície na Figura 4. Essa interação elimina o cruzamento entre esses modos dando origem a um novo modo que possui uma natureza híbrida fóton-plásmon o qual, por essa razão, é denominado Plásmon-Polarítons de Superfície.

Podemos destacar duas regiões na Figura 4. Para valores pequenos de vetor de onda, $k_{x}^{\prime} \rightarrow 0$, a curva de dispersão do SPP está muito proxima da linha da luz. Isto significa que nesta região os SPPs possuem 
um caráter fotônico. A medida que $k_{x}^{\prime}$ aumenta, a curva de dispersão se afasta cada vez mais da linha de luz e se aproxima assintoticamente da frequência $\omega_{S}=\omega_{p l} / \sqrt{2}$ quando $k_{x}^{\prime}>>\omega_{p l} / \sqrt{2} c$.

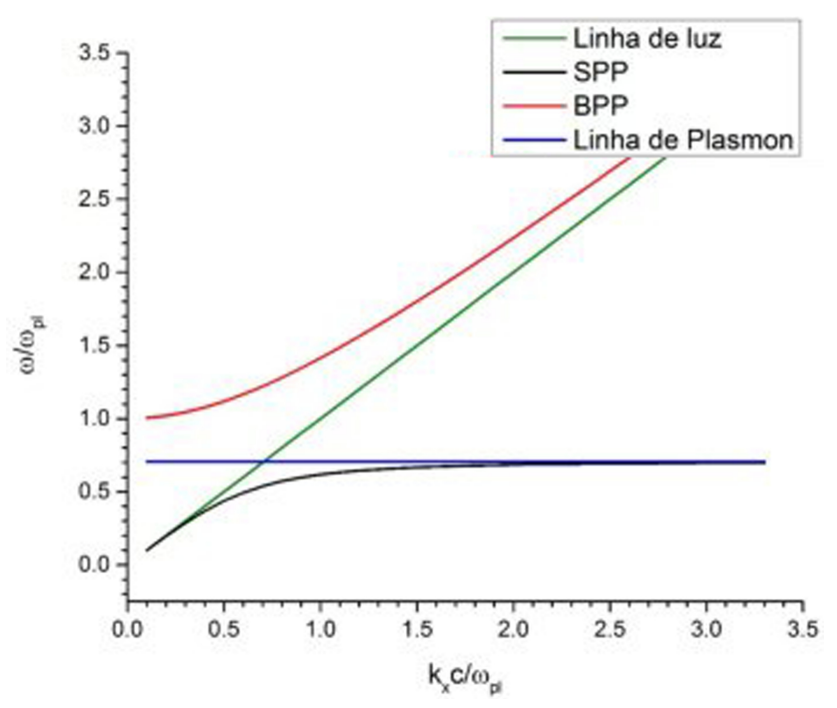

Figura 4: Relações de dispersão do plásmon-poláriton de volume (curva vermelha), para a luz no vácuo (linha verde), o plásmon de superfície (linha azul) e para o plásmon polaríton de superfície (curva em preto).

Na região oposta, $k_{x}^{\prime} \rightarrow \infty$, como já dito, a curva de dispersão tende assintoticamente para o valor $\omega_{S}=\omega_{p l} / \sqrt{2}$ que define a linha do plásmon de superfície. Assim, na região $k_{x}^{\prime} \rightarrow \infty$ o SPP possui um caráter plasmônico. É interessante notar que podemos obter o mesmo resultado a partir da equação (54). Dessa equação vemos que, para $\Gamma=0, k_{x}^{\prime} \rightarrow \infty$ quando a frequência assume o valor

$$
\omega_{S}=\frac{\omega_{p l}}{\sqrt{\epsilon_{\infty}+\epsilon_{2}}},
$$

a qual, para $\epsilon_{\infty}=\epsilon_{2}=1$, assume o valor $\omega_{S}=$ $\omega_{p l} / \sqrt{2}$.

\section{Conclusões}

Neste trabalho realizamos uma introdutória apresentação pedagógica da emergente disciplina da plasmônica, a qual estuda a interação entre uma onda eletromagnética e os elétrons de condução de uma superfície metálica na inteface entre um metal e um dielétrico, incluindo nanoestruturas metálicas. Sob certas condições, essa interação produz o confinamento da luz em dimensões da ordem ou menores que seu comprimento de onda. Aqui, partindo das equações de Maxwell, deduzimos em detalhes a relação de dispersão para o plásmon-poláriton de superfície na interface plana entre um metal e um dielétrico. Conseguimos obter relações gerais, as quais recuperam expressões conhecidas da literatura. Também obtivemos uma expressão explícita para a relação de dispersão, $\omega$ versus $k_{x}$, utilizando a expressão de Drude para a função dielétrica de um gás clássico de elétrons. Adicionalmente, procuramos fazer uma discussão detalhada sobre o significado físico dos resultados obtidos.

\section{Apêndice A}

Como vimos na Introdução, as variadas propriedades físicas de um sólido podem ser descritas em termos do conceito fundamental de quasipartículas ou excitações elementares. A interação entre diferentes tipos de excitações elementares dá origem a novas quasipartículas que resultam do acoplamento entre estas (os modos híbridos ou modos acoplados). Como exemplo típico podemos citar os diferentes tipos de poláritons. Contudo, é importante enfatizar que só pode haver acoplamento entre modos de vibração paralelos, i.e., para haver acoplamento ambos os modos devem ser longitudinais ou ambos devem ser transversais. Assim, o fônon-poláriton resulta do acoplamento entre o fóton e o fônon transversal ótico. Não é possível, por exemplo, haver acoplamento entre o fóton e o fônon longitudinal ótico.

As excitações elementares podem, em grande parte, ser entendidas a partir da análise das equações de Maxwell em um meio material, escrita em termos de componentes de Fourier (ver Apêndice B).

A lei de Gauss, equação (B.16) pode ser escrita como

$$
\epsilon(\vec{k}, \omega) \vec{k} \cdot \vec{E}(\vec{k}, \omega)=0 .
$$

Da equação A.1 temos duas soluções possíveis:

$$
\epsilon(\vec{k}, \omega)=0
$$

$\mathrm{e}$

$$
\vec{k} \cdot \vec{E}(\vec{k}, \omega)=0 .
$$

A primeira solução, equação A.2, está associada as oscilações longitudinais coletivas do plasma que, na linguagem de segunda quantização, constituem os plásmons que são determinados pelas raízes de $\epsilon(\vec{k}, \omega)$. Usando o modelo de Drude e desprezando amortecimento $(\Gamma=0)$, vemos da equação (51) que 
a condição $\epsilon(\vec{k}, \omega)=0$ ocorre para $\omega=\omega_{p l} / \sqrt{\epsilon}$. Como os plásmons de volume correspondem a modos longitudinais, não pode haver acoplamento entre estes e os fótons. A segunda solução, equação A.3., define a condição de transversalidade.

Das equações de Maxwell, na ausência de fontes, obtemos a seguinte equação

$$
\nabla \times(\nabla \times \vec{E})=-\frac{1}{c^{2}} \frac{\partial^{2}}{\partial t^{2}}(\epsilon \vec{E})
$$

a qual, em termos de componentes de Fourier, pode ser escrita como

$$
(\vec{k} \cdot \vec{E}(\vec{k}, \omega)) \vec{k}-k^{2} \vec{E}(\vec{k}, \omega)=-\frac{\omega^{2}}{c^{2}} \epsilon(\vec{k}, \omega) \vec{E}(\vec{k}, \omega)
$$

Utilizando A.3 em A.5 obtemos

$$
k^{2}=\frac{\omega^{2}}{c^{2}} \epsilon(\vec{k}, \omega),
$$

que corresponde à relação de dispersão para o fóton se propagando em um meio material. Vemos que essa propagação é mediada pela interação entre o fóton e os elétrons de condução do material. O fóton revestido por essa interação dá origem a uma nova quasipartícula denominada plásmon-poláriton de volume.

\section{Apêndice B}

As equações de Maxwell em um meio material, utilizando o sistema cgs, são escritas como

$$
\begin{gathered}
\nabla \times \vec{H}(\vec{r}, t)=\frac{1}{c} \frac{\partial \vec{D}(\vec{r}, t)}{\partial t}+\frac{4 \pi}{c} \vec{J}_{c o n}(\vec{r}, t) \\
\nabla \times \vec{E}(\vec{r}, t)=-\frac{1}{c} \frac{\partial \vec{B}(\vec{r}, t)}{\partial t} \\
\nabla \cdot \vec{D}(\vec{r}, t)=4 \pi \rho_{\text {ext }}(\vec{r}, t) \\
\nabla \cdot \vec{B}(\vec{r}, t)=0,
\end{gathered}
$$

onde $\vec{D}(\vec{r}, t)$ é o vetor deslocamento elétrico, $\vec{J}_{c o n}(\vec{r}, t)$ é a densidade de corrente de condução e $\rho_{\text {ext }}(\vec{r}, t)$ é a densidade volumétrica de carga.

Muitas vezes é conveniente escrever as equações de Maxwell em termos de componentes de Fourier.

Assumindo um meio sem dispersão espacial podemos escrever o campo $\vec{E}(\vec{r}, t)$ na seguinte forma

$$
\vec{E}(\vec{r}, t)=\frac{1}{2 \pi} \int_{-\infty}^{\infty} \vec{E}(\vec{r}, \omega) e^{-\imath \omega t} d \omega
$$

onde $\vec{E}(\vec{r}, \omega)$ é a transformada de Fourier de $\vec{E}(\vec{r}, t)$ dada por

$$
\vec{E}(\vec{r}, \omega)=\int_{-\infty}^{\infty} \vec{E}(\vec{r}, \omega) e^{\imath \omega t} d t,
$$

com expresões semelhantes para os outros campos.

Diferenciando o campo elétrico temos que

$$
\nabla \times \vec{E}(\vec{r}, t)=\frac{1}{2 \pi} \int_{-\infty}^{\infty} \nabla \times \vec{E}(\vec{r}, \omega) e^{-\imath \omega t} d \omega
$$

$$
\nabla \cdot \vec{E}(\vec{r}, t)=\frac{1}{2 \pi} \int_{-\infty}^{\infty} \nabla \cdot \vec{E}(\vec{r}, \omega) e^{-\imath \omega t} d \omega
$$

$$
\frac{\partial \vec{E}(\vec{r}, t)}{\partial t}=\frac{1}{2 \pi} \int_{-\infty}^{\infty}(-\imath \omega) \vec{E}(\vec{r}, \omega) e^{-\imath \omega t} d \omega
$$

Usando as equações acima nas equações de Maxwell, estas ficam

$$
\begin{gathered}
\nabla \times \vec{H}(\vec{r}, \omega)=-\frac{\imath \omega}{c} \vec{D}(\vec{r}, \omega)+\frac{4 \pi}{c} \vec{J}_{\text {con }}(\vec{r}, \omega) \\
\nabla \times \vec{E}(\vec{r}, \omega)=\frac{\imath \omega}{c} \vec{B}(\vec{r}, \omega) \\
\nabla \cdot \vec{D}(\vec{r}, \omega)=4 \pi \rho_{\text {ext }}(\vec{r}, \omega) \\
\nabla \cdot \vec{B}(\vec{r}, \omega)=0
\end{gathered}
$$

Para um meio não-magnético $(\vec{B}=\vec{H})$ e na ausência de fontes temos que

$$
\begin{gathered}
\nabla \times \vec{H}(\vec{r}, \omega)=-\frac{\imath \omega}{c} \epsilon(\omega) \vec{E}(\vec{r}, \omega) \\
\nabla \times \vec{E}(\vec{r}, \omega)=\frac{\imath \omega}{c} \vec{H}(\vec{r}, \omega) \\
\nabla \cdot \epsilon(\omega) \vec{E}(\vec{r}, \omega)=0 \\
\nabla \cdot \vec{H}(\vec{r}, \omega)=0
\end{gathered}
$$

onde usamos que $\vec{D}(\vec{r}, \omega)=\epsilon(\omega) \vec{E}(\vec{r}, \omega)$. 


\section{Agradecimentos}

A.V. A-N agradece à Coordenação de Aperfeiçoamento de Pessoal de Nível Superior (CA$\mathrm{PES}$ ) pelo suporte financeiro para o desenvolvimento deste trabalho (proc. 23038.003304/2016-72). A. R$\mathrm{N}$. agradece ao $\mathrm{CNPq}$ pela bolsa de iniciação científica. A. J. agradece financiamento do $\mathrm{CNPq}$ (552124/2011-7) e FINEP (01.13.0330.00).

\section{Referências}

[1] Marvin L. Cohen, Phys. Rev. Lett. 101, 250001 (2008).

[2] Ado Jorio, Bioengineering Applications of Carbon Nanostructures (Springer Verlag, Berlin, 2016).

[3] M. Dragoman and D. Dragoman, Progress in Quantum Electronic 32, 1 (2008).

[4] Ricardo S. Oyarzabal, José D. Szezech Junior, Antonio M. Batista, Iberê L. Caldas, Ricardo L. Viana e Kelly C. Iarosz, Revista Brasileira de Ensino de Física 37, 2308 (2015).

[5] P.M. Platzman and P.A. Wolff, in: Solid State Physics, Suppl. 13, edited by F. Seitz, D. Turnbull and H. Ehrenrich (Academic Press, New York, 1973).

[6] Lukas Novotny and Bert Hecht, Principles of NanoOptics (Cambridge University Press, Cambridge, 1999), $2^{\mathrm{a}}$ ed, p. 5, p. 371.

[7] Rodrigo Gonçalves Pereira e Eduardo Miranda, Revista Brasileira de Ensino de Física 24, 237 (2002).
[8] Indianara Silva, Revista Brasileira de Ensino de Física 37, 4204 (2015).

[9] Richard D. Mattuck, A Guide to Feynman Diagrams in the Many-body Problem (McGraw-Hill, New York, 1967).

[10] S. Nakajima, Y. Toyozawa and R. Abe, The Physics of Elementary Excitations (Springer-Verlag, Berlin, 1980).

[11] Harry A. Atwater, Scientific American 296, 56 (2007).

[12] Francisco E.G. Guimarães, Revista Brasileira de Ensino de Física 18, 56 (1996).

[13] Daniel M. Reis, Edson M. Santos e A.V. AndradeNeto, Revista Brasileira de Ensino de Física 37, 2312 (2015).

[14] Thiago L. Vasconcelos, Bráulio S. Archanjo, Benjamin Fragneaud, Bruno S. Oliveira, Juha Riikonen, Changfeng Li, Douglas S. Ribeiro, Cassiano Rabelo, Wagner N. Rodrigues, Ado Jorio, et. al., ACSNano 9, 6297 (2015).

[15] John R. Reitz, Frederick J. Milford e Robert W. Christy, Fundamentos da Teoria Eletromagnética. (Editora Campus, São Paulo, 1982), p. 95, p. 199.

[16] Stefan Alexander Maier, Plasmonics: Fundamentals and Applications (Springer, New York, 2007), p. 10-27.

[17] H. Raether, Surface Plasmons on Smooth and Rough Surfaces and on Gratings (Springer-Verlag, Berlin, 1998), Appendix I.

[18] William L. Barnes, J. Opt. A: Pure Appl. Opt. 8, S87 (2006).

[19] A.V. Andrade-Neto, Revista Brasileira de Ensino de Física 39, e2304 (2017). 